\title{
BMJ Open Acupuncture for adults with overactive bladder: a systematic review protocol
}

\author{
Qian Mo, ${ }^{1,2}$ Yang Wang, ${ }^{1}$ Yongming Ye, ${ }^{1}$ Jinna Yu, ${ }^{1}$ Zhishun Liu ${ }^{1}$
}

To cite: Mo Q, Wang Y, Ye Y, et al. Acupuncture for adults with overactive bladder: a systematic review protocol. BMJ Open 2015;5:e006756. doi:10.1136/bmjopen-2014006756

- Prepublication history for this paper is available online. To view these files please visit the journal online (http://dx.doi.org/10.1136/ bmjopen-2014-006756).

QM and YW contributed equally.

Received 27 September 2014 Accepted 25 November 2014

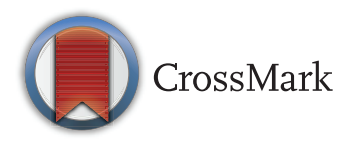

${ }^{1}$ Department of Acupuncture, Guang'anmen Hospital, China Academy of Chinese Medical Sciences, Beijing, China

${ }^{2}$ School of Graduates, Beijing University of Chinese Medicine, Beijing, China

Correspondence to Dr Zhishun Liu; liuzhishun@aliyun.com

\section{ABSTRACT}

Introduction: Overactive bladder $(O A B)$ is a symptom syndrome defined by the International Continence Society (ICS) as 'the presence of urinary urgency (both daytime and nighttime), usually accompanied by increased frequency and nocturia with or without urge urinary incontinence in the absence of a urinary tract infection or other obvious pathology'. Clinical studies indicate that acupuncture could reduce micturition over $24 \mathrm{~h}$, urgency episodes over $24 \mathrm{~h}$, and improve quality of life among people with OAB. This systematic review protocol details the proposed methods for evaluating the effectiveness and safety of acupuncture for OAB.

Methods and analysis: The following databases will be searched for relevant studies: the Cochrane Central Register of Controlled Trials (CENTRAL), the Cochrane Incontinence Group Trials Register, MEDLINE, EMBASE, Chinese Biomedical Literature Database (CBM), Chinese Medical Current Content (CMCC), Chinese Scientific Journal Database (VIP database), Wan-Fang Data, and China National Knowledge Infrastructure (CNKI) and will hand search a list of medical journals as a supplement. Any randomised controlled trials in English or Chinese without restriction of publication status will be included with treatment of OAB. Outcomes will mainly include number of micturition episodes over $24 \mathrm{~h}$, number of urgency episodes over $24 \mathrm{~h}$ and number of incontinence episodes over $24 \mathrm{~h}$. Two reviewers will independently screen the titles, abstracts or even full texts, and extract data. Two other reviewers will assess study quality. Revman 5.1 software will be used to conduct meta-analysis and calculate the risk ratio for dichotomous data. Weighted mean difference or standard mean difference will be calculated for continuous data. The Cochrane collaboration's tool will be used to assess the risk of bias.

Dissemination: This systematic review protocol will provide information on acupuncture therapy for $\mathrm{OAB}$. The results will be disseminated through peer-reviewed publication or conference presentations.

Protocol registration: PROSPERO CRD42014010181.

\section{INTRODUCTION}

\section{Description of the condition}

Overactive bladder $(\mathrm{OAB})$ is a symptom syndrome defined by the International
Continence Society (ICS) as 'the presence of urinary urgency (both daytime and nighttime), usually accompanied by increased frequency and nocturia with or without urge urinary incontinence in the absence of a urinary tract infection or other obvious pathology'. ${ }^{2} \mathrm{OAB}$ has a serious impact on the quality of life of patients. ${ }^{3}{ }^{4}$ It can cause anxiety and depression, and it influences patients' social interactions and sexual function. ${ }^{3-6}$

\section{Description of the intervention}

Acupuncture is a branch of traditional Chinese medicine (TCM) that encompasses many methods, such as body acupuncture, scalp acupuncture, auricular acupuncture, warm needling, fire needling and elongated needling. Acupuncture has been used to treat urinary incontinence since ancient times in China. The HuangdiNeijing (The Yellow Emperor's Canon of Medicine) says 'For needling, the reinforcing methods should be used for urinary incontinence and the reducing method for urinary retention' ${ }^{7}$

\section{How the intervention might work}

Acupuncture works by stimulating points with needles. In TCM theory, acupuncture is thought to regulate qi circulation. For OAB, needling at points on the kidney or bladder meridians could reinforce qi and promote the recovery of bladder function. ${ }^{8} 9$ In Western medicine, the mechanism of acupuncture is as yet unknown.

\section{Why it is important to perform this review}

The American Urological Association (AUA) recommends a three-line therapy for $\mathrm{OAB} .{ }^{3}$ The first-line therapy is behavioural therapy, such as delayed voiding bladder training, pelvic floor muscle training, lifestyle modifications and management of fluid intake. These therapies are suitable for all $\mathrm{OAB}$ patients; however, patients need to invest much time and effort to achieve good effects. ${ }^{10-12}$ The second line of therapy is 
oral anticholinergic drugs such as tolterodine, solifenacin and oxybutynin. Although drug therapy is noninvasive, side effects such as dry mouth, constipation, headache and vision abnormality may affect quality of life. $^{12-15}$ The third line of therapy refers to neural regulation such as sacral nerve stimulation; however, this treatment is limited because it is invasive and can cause adverse reactions such as infection. ${ }^{3} 16$

Acupuncture is an alternative therapy for OAB; it is minimally invasive and causes few side effects. ${ }^{17-20}$ Several clinical studies have shown that acupuncture could reduce micturition over $24 \mathrm{~h}$, urgency episodes over $24 \mathrm{~h}$, and improve quality of life. ${ }^{21-25}$ Two systematic reviews have also been found on acupuncture for urinary incontinence and stress urinary incontinence. ${ }^{8} 9$ Both reviews reported favourable effects of acupuncture on symptoms, but neither of them determined its effect for insufficient evidence. No systematic review has been found about acupuncture for $\mathrm{OAB}$; it is therefore important to establish whether acupuncture is a good choice for $\mathrm{OAB}$ patients who do not want surgery, and whether it is as effective as other therapies.

The objective of this proposed systematic review is to determine the effectiveness and safety of acupuncture for $\mathrm{OAB}$ in adults. The following comparisons will be addressed:

1. Acupuncture versus placebo or no treatment.

2. Acupuncture versus any other treatment.

\section{METHODS}

Criteria for included studies

Type of studies

Randomised controlled trials (RCTs) in English or Chinese will be included without restriction of publication type. Quasi-RCTs will be excluded as they are not truly randomised, and there is a greater risk of selection bias in trials in which allocation is not adequately concealed.

\section{Type of participants}

Participants of any race or gender with a diagnosis of $\mathrm{OAB}$ (according to the definition of the ICS and the guidelines of the $\mathrm{AUA}^{3}$ ) will be included. Participants must be at least 18 years old.

\section{Type of interventions}

The following acupuncture methods will be included: body acupuncture, electro-acupuncture, scalp acupuncture, warm acupuncture, elongated needling, and auricular acupuncture. The control interventions of included studies may include placebo, sham acupuncture (minimal, non-point), waiting list, no treatment, and any active treatment ${ }^{3}$ (ie, bladder retraining, pharmacological therapies, neurologic stimulation, surgery).

Laser acupuncture, dry needling, cupping and acupressure will be excluded. Laser acupuncture and dry needling are not routine or traditional acupuncture methods, and cupping and acupressure are different therapies from acupuncture in TCM. ${ }^{9}$ Studies with the following comparisons will also be excluded:

1. Acupuncture plus another therapy versus the same other therapy plus drug therapy

2. Acupuncture plus a therapy versus another different therapy

3. One kind of acupuncture versus another kind of acupuncture

Studies comparing acupuncture plus a therapy versus the same therapy will be included.

\section{Type of outcome measures}

Primary outcomes

1. Number of micturition episodes over $24 \mathrm{~h}$

2. Number of urgency episodes over $24 \mathrm{~h}$

3. Number of incontinence episodes over $24 \mathrm{~h}$

Secondary outcomes:

1. Pads used over $24 \mathrm{~h}$

2. Number of nocturnal awakenings related to $\mathrm{OAB}$ over $24 \mathrm{~h}$

3. Maximum cystometric capacity $(\mathrm{mL})$

4. Quality of life (quality of life assessment using any of the available validated instruments, such as Distress Inventory (UDI-6), the Incontinence Impact Questionnaire (IIQ-7), ${ }^{26}$ OAB Questionnaire (OAB-q) ${ }^{27}$ and the Incontinence Quality of Life Scales (IQOL) ${ }^{28}$ )

5. Safety (measured by incidence and severity of adverse effects)

\section{Search methods for the identification of studies}

A search strategy will be designed and conducted according to the Cochrane handbook guidelines. ${ }^{29}$

\section{Electronic searches}

We will search the following databases from inception to July 2014:

1. The Cochrane Incontinence Group Trials Register

2. MEDLINE

3. EMBASE

4. The Cochrane Central Register of Controlled Trials (CENTRAL)

5. Chinese Biomedical Literature Database (CBM)

6. Chinese Medical Current Content (CMCC)

7. China National Knowledge Infrastructure (CNKI)

8. Wan-Fang Data

9. Chinese Scientific Journal Database (VIP)

Table 1 presents the full list of search terms to be used.

\section{Searching other resources}

The following medical journals published out of the time span of the databases will be manually searched in libraries: Acupuncture Research (1976-July 1998), Chinese Acupuncture and Moxibustion (1981-July 1998), Journal of Clinical Acupuncture and Moxibustion (1985-July 1998) and Shanghai Journal of Acupuncture and Moxibustion (1982-July 1998). 


\section{Table 1 Ovid MEDLINE search strategy}

\begin{tabular}{ll}
\hline $\mathbf{N}$ & Search items \\
\hline 1 & randomized controlled trial.pt. \\
2 & controlled clinical trial.pt. \\
3 & randomized.ab. \\
4 & randomly.ab. \\
5 & placebo.ab. \\
6 & drug therapy.fs. \\
7 & trial.ab. \\
8 & groups.ab. \\
9 & or/1-8. \\
10 & exp animals/ not humans.sh. \\
11 & 9 not 10 \\
12 & exp acupuncture therapy. \\
13 & acupuncture.mp. \\
14 & acupressure.tw. \\
15 & (acupoint or electroacupuncture or \\
& electro-acupuncture).mp. \\
16 & elongated needle.mp. \\
17 & warming needle. \\
18 & auricular acupuncture. \\
19 & or/12-18. \\
20 & OAB.tw. \\
21 & (overactive bladder or overactive bladder syndrome or \\
& bladder overactive or urge inconvenience or \\
& overactive detrusor or urinary bladder, overactive or \\
& detrusor overactivity, bladder instability, detrusor \\
22 & instability).tw. \\
23 & or/20-21 and 19 and 22 \\
\hline
\end{tabular}

This search strategy will be suitable for other electronic databases.

In addition, for studies without the full text, we will try to contact the authors for the full text.

\section{Data collection and analysis}

Selection of studies

Two reviewers (QM and YW) will screen the studies at the same time independently. If any disagreement occurs, a decision will be made through discussion or consultation with a third author (ZL). Details of the study selection procedure are shown in figure 1 . Excluded studies will be listed in a table with reasons.

\section{Data extraction}

Before data extraction, a standard form containing specified outcomes will be created after discussion among all reviewers. Two reviewers (QM and YW) will extract data independently using this standard form. When data in included studies have been collected but not reported, reviewers will seek clarification from the author. If the reviewers have different opinions, the issue will be resolved through discussion or consultation with a third author (ZL).

\section{Risk of bias assessment}

The reviewers will independently assess six domains of bias (sequence generation, allocation concealment,

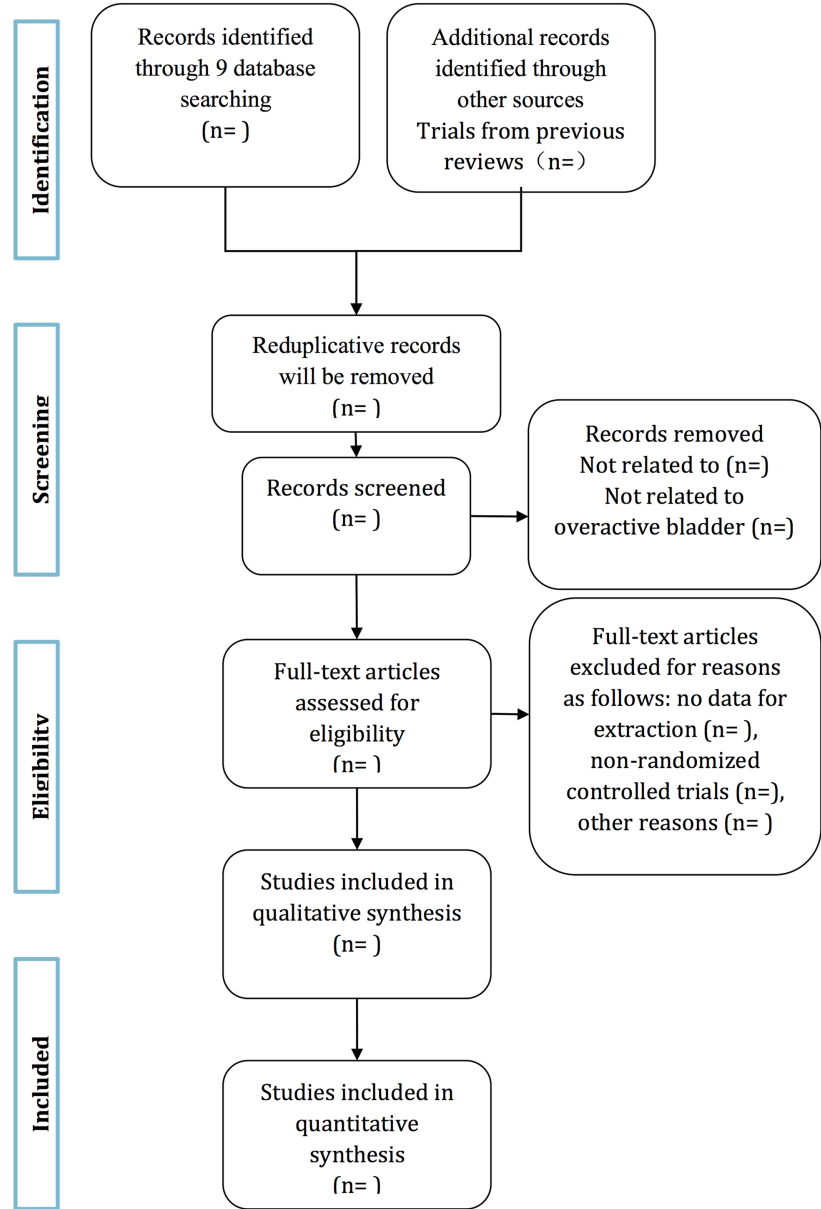

Figure 1 Process of the systematic review.

blinding or masks, incomplete data assessment, selective outcome reporting, and other sources of bias). ${ }^{29}$ The studies will then be classified into three levels of bias: low, unclear, and high risk of bias. Differences in opinion will be resolved by discussion or consultation with a third author (ZL).

\section{Measures of treatment effect}

Analysis will be based on available data of included studies. For dichotomous data, the risk ratio (RR) will be calculated with $95 \%$ CI. For continuous variables, we will use means and SDs to calculate a mean difference (MD) with $95 \%$ CI.

\section{Unit of analysis issues}

The unit of analysis is the individual participant (unit to be randomised for interventions to be compared).

\section{Dealing with missing data}

For studies with missing data, the reviewers will try to obtain the information by contacting the study investigators. If the investigators cannot be contacted, we will base our analysis on the available data.

\section{Assessment of heterogeneity}

Higgins $\mathrm{I}^{2}$ statistics will be used to test inconsistencies among the included trials. The cut off point of $\mathrm{I}^{2}$ 
statistics is $50 \% .{ }^{29} \mathrm{I}^{2}>50 \%$ will indicate the existence of significant heterogeneity among studies. ${ }^{29}$ In this case, a meta-analysis will be conducted to find the source of heterogeneity. Subgroup analysis will be conducted according to the source of heterogeneity.

\section{Assessment of reporting biases}

A funnel plot will be used to evaluate publication bias if more than 10 studies are included.

\section{Data synthesis}

Revman 5.1 software will be used to conduct meta-analysis and calculate the RR, and 95\% CI for dichotomous data. Weighted mean difference (WMD) or standard mean difference (SMD) and 95\% CI will be calculated for continuous data. If the same outcome measurement tool and unit was used, the WMD and 95\% CI will be calculated, or otherwise the SMD and 95\% CI. If the included studies have existing heterogeneity (the $p$ value is $<0.05$ ), the RR, WMD or SMD will be calculated by the random-effect model. Otherwise, a fixed-effect model will be used.

\section{Subgroup analysis and investigation of heterogeneity}

There is no pre-subgroup plan. If data are available, factors like acupuncture methods, existence of detrusor overactivity (DO) and sex of patients will be taken into account.

\section{Sensitivity analysis}

The sensitivity analysis will be used to assess whether the sample size and missing data impact the results of this review. If there are adequate studies (no less than three studies), we will conduct a sensitivity analysis to check the robustness of conclusions and assess the impact of methodological quality.

\section{Ethics and dissemination}

This review does not need ethical approval, as data used here are not individual or private. The results will provide a general overview and evidence of effectiveness and safety of acupuncture for OAB. They will also have implications for clinical practice and further research.

\section{DISCUSSION}

Studies have shown that acupuncture is effective for relieving the symptoms of $\mathrm{OAB}$; however, its effect has not been scientifically evaluated. ${ }^{21-25}$ We present a protocol of a systematic review to determine the effectiveness and safety of acupuncture for OAB. The conclusions drawn from this review will benefit policy makers, patients and clinicians seeking effective and minimally invasive methods for treating $\mathrm{OAB}$.

This study might have some limitations. Specifically, different forms of acupuncture therapies and qualities of methodology may cause significant heterogeneity. Because of the language barrier, only English and
Chinese medical database will be included. Hence, some relevant studies in other languages might be missed.

Contributors QM and YW contributed to the conception of the study. The manuscript of the protocol was drafted by $\mathrm{QM}$ and $\mathrm{YW}$, and was revised by ZL. QM and YW will also independently screen the potential studies and extract data from included studies. JY and YY will assess the risk of bias, and finish data synthesis. ZL will arbitrate any disagreements and ensure that no errors occur during the review. All authors have approved the publication of the protocol.

Funding This research received no specific grant from any funding agency in the public, commercial or not-for-profit sectors.

Competing interests None.

Provenance and peer review Not commissioned; externally peer reviewed.

Open Access This is an Open Access article distributed in accordance with the Creative Commons Attribution Non Commercial (CC BY-NC 4.0) license, which permits others to distribute, remix, adapt, build upon this work noncommercially, and license their derivative works on different terms, provided the original work is properly cited and the use is non-commercial. See: http:// creativecommons.org/licenses/by-nc/4.0/

\section{REFERENCES}

1. Wein AJ, Rovner ES. Definition and epidemiology of overactive bladder. Urology 2002;60(5 Suppl):7-12.

2. Haylen BT, de Ridder D, Freeman RM, et al. An International Urogynecological Association (IUGA)/International Continence Society (ICS) joint report on the terminology for female pelvic floor dysfunction. Neurourol Urodyn 2010;29:4-20.

3. Gormley EA, Lightner DJ, Burgio KL, et al. Diagnosis and Treatment of Overactive Bladder (Non-Neurogenic) in Adults: AUA/SUFU Guideline. J Urol 2012;188(6 Suppl):2455-63.

4. Al-Ghazo MA, Ghalayini IF, Al-Azab R, et al. Urodynamic Detrusor Overactivity in Patients with Overactive Bladder Symptoms. Int Neurourol J 2011;15:48-54.

5. Winters JC, Dmochowski RR, Goldman HB, et al. Urodynamic studies in adults: AUA/SUFU guidelines. J Urol 2012;188(6 Suppl):2464-72

6. Sexton CC, Coyne KS, Thompson C, et al. Prevalence and effect on health-related quality of life of overactive bladder in older Americans: results from the epidemiology of lower urinary tract symptoms study. J Am Geriatr Soc 2011;59:1465.

7. Zhang L, Yang Y, Liu HL, et al. Urinary incontinence in ancient Chinese Medical literature. J Tradit Chin Med Lit 2013;2:54-6.

8. Sun-Ho P, Su-Ryun H, Oh-Jun K, et al. Acupuncture for the treatment of urinary incontinence: A review of randomized controlled trials. Exp Therap Med 2013;6:773-80.

9. Wang $\mathrm{Y}$, Zhishun L, Peng W, et al. Acupuncture for stress urinary incontinence in adults. Cochrane Database Syst Rev 2013;7: CD009408.

10. Burgio KL, Goode PS, Johnson TM II, et al Behavioral versus drug treatment for overactive bladder in men: the male overactive bladder treatment in veterans (MOTIVE) trial. J Am Geriatr Soc 2011;59:2209.

11. Borello-France D, Burgio KL, Goode PS, et al. Urinary incontinence treatment: Adherence to behavioral interventions for urge incontinence when combined with drug therapy: Adherence rates, barriers, and predictors. Phys Ther 2010;90:1493.

12. Hartmann KE, McPheeters ML, Biller DH, et al. Treatment of overactive bladder in women. Evid Rep Technol Assess (Full Rep) 2009;187:1-120, v.

13. Staskin DR, Oefelein MG. Re: Giacomo Novara, Antonio Galfano, Silvia Secco, et al. A Systematic Review and Meta-Analysis of Randomized Controlled Trials with Antimuscarinic Drugs for Overactive Bladder. Eur Urol 2008;54:740-64. Eur Urol 2009;55: e84-85.

14. Maman K, Aballea S, Nazir J, et al. Comparative Efficacy and Safety of Medical Treatments for the Management of Overactive Bladder: A Systematic Literature Review and Mixed Treatment Comparison. Eur Urol 2014;65:755-65.

15. Buser N, Ivic S, Kessler TM, et al. Efficacy and adverse events of antimuscarinics for treating overactive bladder: network meta-analyses. Eur Urol 2012;62:1040-60. 
16. Tang $\mathrm{H}$, Chen $\mathrm{J}$, Wang $\mathrm{Y}$, et al. Combination of sacral neuromodulation and tolterodine for treatment of idiopathic overactive bladder in women: a clinical trial. J Urol 2012;11:1800-5.

17. Emst $E$, White AR. Prospective studies of the safety of acupuncture: a systematic review. Am J Med 2001;110:481-5.

18. Park J, Sohn Y, White AR, et al. The safety of acupuncture during pregnancy: a systematic review. Acupunct Med 2014;32:257-66.

19. Adams D, Cheng F, Jou $\mathrm{H}$, et al. The safety of pediatric acupuncture: a systematic review. Pediatrics 2011;128:e1575-87.

20. Jaung-Geng L, Yi-Hung C, Xin-Yan G, et al. Clinical efficacy, mechanisms, and safety of acupuncture and moxibustion. Evid Based Complement Alternat Med 2014;2014:356258.

21. Bschleipfer T, Lüdecke G, Durschnabel M, et al. Auricular acupuncture in patients with detrusor overactivity: A pilot study. Urologe A 2013;20:1-5.

22. Emmons SL and Otto $\mathrm{L}$. Acupuncture for overactive bladder: a randomized controlled trial. Obstet Gynecol 2005;106:138-43.

23. Kitakoji $\mathrm{H}$, Terasaki T, Honjo $\mathrm{H}$, et al. Effect of acupuncture on the overactive bladder. Nihon Hinyokika Gakkai Zasshi 1995;86:1514-19.
24. Engberg S, Cohen S, Sereika SM. The efficacy of acupuncture in treating urge and mixed incontinence in women: a pilot study. $J$ Wound Ostomy Continence Nurs 2009;36:661-70.

25. Chang KK, Wong TK, Wong TH, et al. Effect of acupressure in treating urodynamic stress incontinence: a randomized controlled trial. Am J Chin Med 2011;39:1139-59.

26. Uebersax JS, Wyman JF, Shumaker SA, et al. Short forms to assess life quality and symptom distress for urinary incontinence in women: the Incontinence Impact Questionnaire and the Urogenital Distress Inventory. Continence Program for Women Research Group. Neurourol Urodyn 1995;14:131-9.

27. Coyne K, Revicki D, Hunt T, et al. Psychometric validation of an overactive bladder symptom and health-related quality of life questionnaire: the OAB- q. Qual Life Res 2002;11:563-74.

28. Patrick DL, Martin ML, Bushnell DM, et al. Quality of life of women with urinary incontinence: further development of the incontinence quality of life instrument (I-QOL). Urology 1999;53:71-6.

29. Higgins JPT, Green S. Cochrane handbook for systematic reviews of interventions version 5.1. 0 [updated March 2011]. The Cochrane Collaboration, 2011. 